(HAPO) study. ${ }^{3,4}$ Fifteen institutional review boards approved our protocol, and none rejected the trial on ethical grounds. In addition, an independent data and safety monitoring committee regularly reviewed the trial. Some participants would not even have met the criteria for gestational diabetes (more evidence of clinical equipoise). We strongly disagree with an assertion that we unnecessarily exposed participants to harm. Randomized trials are necessary provided that there is sufficient uncertainty and that the results will resolve the dispute among physicians, which was the case for the treatment of mild gestational diabetes. ${ }^{5}$ We agree that our results may encourage previously reluctant physicians to treat gestational diabetes, but such treatment is now supported by evidence from a clinical trial rather than by expert opinion alone.

Mark B. Landon, M.D.

Ohio State University College of Medicine

Columbus, $\mathrm{OH}$
Catherine Y. Spong, M.D.

Eunice Kennedy Shriver National Institute of Child Health and Human Development

Bethesda, MD

Elizabeth Thom, Ph.D.

George Washington University

Washington, DC

Since publication of their article, the authors report no further potential conflict of interest.

1. Weight gain during pregnancy: reexamining the guidelines. Washington, DC: National Academies Press, 2009.

2. Screening for gestational diabetes mellitus: U.S. Preventive Services Task Force recommendation statement. Ann Intern Med 2008;148:759-65.

3. Crowther CA, Hiller JE, Moss JR, McPhee AJ, Jeffries WS, Robinson JS. Effect of treatment of gestational diabetes mellitus on pregnancy outcomes. N Engl J Med 2005;352:2477-86.

4. The HAPO Study Cooperative Research Group. Hyperglycemia and adverse pregnancy outcomes. N Engl J Med 2008;358: 1991-2002.

5. Holt RIG. The Hyperglycemia and Adverse Pregnancy Outcomes trial: answers but still more questions about the management of gestational diabetes. Diabet Med 2008;25:1013-4.

\title{
Dectin-1 Deficiency and Mucocutaneous Fungal Infections
}

TO THE EDITOR: Mucocutaneous candidiasis is commonly seen in persons with deficient T-cellmediated immunity, including newborn infants, patients with the acquired immunodeficiency syndrome, and those with genetically defined primary T-cell deficiencies. ${ }^{1}$ In their Brief Report about human dectin-1 deficiency and mucocutaneous fungal infections, Ferwerda et al. (Oct. 29 issue) ${ }^{2}$ suggest that chronic mucocutaneous candidiasis may also be caused by a genetic defect of the $\beta$-glucan receptor dectin- 1 . However, their data should be interpreted with caution. First, they provide no information about how dectin-1 deficiency impairs overall T-cell-dependent mucosal immunity against candida. Second, they suggest that the lack of invasive fungal infection in dectin-1-deficient patients may be attributed to the normal phagocytosis and killing of candida by granulocytes. This explanation sounds reasonable but conflicts with observations of invasive microbial disease in patients with other, recently described innate immune deficiencies. ${ }^{3,4}$ Finally, it is difficult to define the precise immunologic role of dectin- 1 in immunity to candida in light of the demonstration by Saijo et al. ${ }^{5}$ that dectin- 1 may not actually be required for host defense against Candida albicans.

\author{
László Maródi, M.D. \\ Melinda Erdös, M.D. \\ University of Debrecen \\ Debrecen, Hungary \\ Imarodi@dote.hu
}

No potential conflict of interest relevant to this letter was reported.

1. Maródi L. Local and systemic host defense mechanisms against Candida: immunopathology of candidal infections. Pediatr Infect Dis J 1997;16:795-801.

2. Ferwerda B, Ferwerda G, Plantinga TS, et al. Human dectin-1 deficiency and mucocutaneous fungal infections. N Engl J Med 2009;361:1760-7.

3. Ku CL, Picard C, Erdös M, et al. IRAK-4 and NEMO mutations in otherwise healthy children with recurrent invasive pneumococcal disease. J Med Genet 2007;44:16-23.

4. von Bernuth H, Picard C, Jin Z, et al. Pyogenic bacterial infections in humans with MyD88 deficiency. Science 2008;321: 691-6.

5. Saijo S, Fujikado N, Furuta T, et al. Dectin-1 is required for host defense against Pneumocystis carinii but not against Candida albicans. Nat Immunol 2007;8:39-46.

THE AUTHORS REPLY: In response to Maródi and Erdös: T-cell-derived immunity, and especially responses of type 17 helper $\mathrm{T}$ cells (Th17), are crucial for mucosal antifungal defense. ${ }^{1,2}$ Our report describes deficient Th17-cell responses in patients with dectin- 1 deficiency, ${ }^{3}$ and a similar defect has been reported in persons with defective 
CARD9, the caspase recruitment domain-containing protein $9 .{ }^{3}$ The fact that dectin-1 deficiency increases susceptibility to mucocutaneous, but not systemic, fungal infections stresses the specific role that dectin-1 may have in mucosal antifungal host defense. This is not in conflict with a role of NEMO (the inhibitor of kappa light polypeptide gene enhancer in B cells, kinase gamma), IRAK-4 (the interleukin-1 receptor-associated kinase 4), or MyD88 (the protein encoded by myeloid differentiation primary response gene 88 ) for the host defense against systemic bacterial or fungal infections, since these are adaptor molecules specific for toll-like receptors, a different class of pattern-recognition receptors. ${ }^{4}$ The suggestion by Saijo et al. ${ }^{5}$ in their study that dectin-1 is not involved in the host defense against systemic candidiasis in mice reinforces the conclusion that dectin- 1 may be specific for mucosal antifungal defense.
Gerben Ferwerda, Ph.D., M.D.

Bart Jan Kullberg, Ph.D., M.D.

Mihai G. Netea, Ph.D., M.D.

Radboud University Nijmegen Medical Center

Nijmegen, the Netherlands

m.netea@aig.umcn.nl

Since publication of their article, the authors report no further potential conflict of interest.

1. Conti HR, Shen F, Nayyar N, et al. Th17 cells and IL-17 receptor signaling are essential for mucosal host defense against oral candidiasis. J Exp Med 2009;206:299-311.

2. Milner JD, Brenchley JM, Laurence A, et al. Impaired T(H)17 cell differentiation in subjects with autosomal dominant hyperIgE syndrome. Nature 2008;452:773-6.

3. Glocker E-O, Hennigs A, Nabavi M, et al. A homozygous CARD9 mutation in a family with susceptibility to fungal infections. N Engl J Med 2009;361:1727-35.

4. Netea MG, Van Der Graaf CA, Vonk AG, Verschueren I, Van Der Mer JW, Kullberg BJ. The role of toll-like receptors (TLR) 2 and TLR4 in the host defense against disseminated candidiasis. J Infect Dis 2002;185:1483-9.

5. Saijo S, Fujikado N, Furuta T, et al. Dectin-1 is required for host defense against Pneumocystis carinii but not against Candida albicans. Nat Immunol 2007;8:39-46.

\section{Taxing Sugar-Sweetened Beverages}

TO THE EDITOR: Brownell et al. (Oct. 15 issue) ${ }^{1}$ provide support for a tax on sugar-sweetened beverages in part by citing the results of longterm, randomized, controlled trials. They cite a report of a 1-year trial involving students 7 to 11 years of age that showed a lower incidence of obesity in the dietary intervention group, although the difference in body-mass index was not significant. ${ }^{2}$ Follow-up 2 years after completion of the trial showed that the difference in the incidence of obesity was not sustained. ${ }^{3}$ This dietary intervention apparently only had a transient effect without affecting the long-term propensity for obesity. None of the three other long-term, randomized, controlled trials cited in the article met their primary end points; an analysis of a different subgroup within each trial was made in an attempt to show some benefit.

The essential failure of these trials should give us pause. Before assigning blame for the obesity epidemic, we should have clinical evidence that an intervention to reduce the consumption of sugar-sweetened beverages is effective in achieving this goal, is either more effective or additive to the effect of other proven dietary therapies, and will reduce the long-term propensity for obesity.
Michael G. Kaplan, M.D.

Maimonides Medical Center Brooklyn, NY

No potential conflict of interest relevant to this letter was reported.

1. Brownell KD, Farley T, Willett WC, et al. The public health and economic benefits of taxing sugar-sweetened beverages. N Engl J Med 2009;361:1599-605.

2. James J, Thomas P, Cavan D, Kerr D. Preventing childhood obesity by reducing consumption of carbonated drinks: cluster randomised controlled trial. BMJ 2004;328:1237.

3. James J, Thomas P, Kerr D. Preventing childhood obesity: two year follow-up results from the Christchurch Obesity Prevention Programme in Schools (CHOPPS). BMJ 2007;335:762.

TO THE EDITOR: Although their intent is unquestionably noble, Brownell and coauthors do not produce an economic rationale for a soda tax; they merely present one side of the economic equation (cost-harm) without consideration of its usefulness. To maintain liberty, we defer to individual persons to balance the cost-utility equation. However, the authors point to market failure as a justification for intervention. Their first two rationales involve the argument that the population lacks the capacity to make free economic decisions. In attempting to restrict peoples' liberty, the onus is on the authors to convincingly 